\title{
A rare case of oesophageal variceal bleed secondary to dialysis catheter-induced superior vena cava stenosis and thrombosis
}

\author{
Authors: Arun Prasath Perumal Thiagarajan, Asmaa Al-Chidadi, Sourjya Kar, Frieder Kleemann, Soubhik Pal, \\ Baharah Arsalanizadeh and Brandon Krijgsman
}

\section{Introduction}

We are presenting a case of bleeding oesophageal varices secondary to superior vena cava stenosis caused by a permanent dialysis line.

\section{Case report}

A 67-year-old female with chronic kidney disease (CKD) stage $\mathrm{V}$ on haemodialysis, secondary to chronic pyelonephritis and secondary focal segmental glomerulosclerosis (FSGS). She started haemodialysis via a permanent internal jugular line in September 2013, which was removed in August 2014 following creating a left brachiocephalic fistula. This fistula was used until it was painful to needle due to hitting a nerve; therefore, a right brachiocephalic arteriovenous fistula (AVF) was fashioned on the right arm in April 2017. The left-sided fistula was left patent.

In May 2018, she complained of progressive worsening purplecoloured lumps over her trunks which were actually extensive varicose veins. For this she was referred to vascular surgical team to rule out central venous thrombosis.

In June 2018, she was admitted with large-volume haematemesis and hypovolemic shock. After volume replacement with blood, an emergency endoscopy showed four columns of Grade II-III varices at the gastro-oesophageal junction. This was banded and the bleeding stopped. She then required another endoscopy due to a further drop in her haemoglobin, which showed further varices that were also banded. A computed tomography (CT) showed a superior vena cava stenosis and a superadded thrombus extending into the azygos vein and down into the left brachiocephalic fistula, together with extensive collateral formation. Both CT and fibroscan didn't demonstrate liver cirrhosis. The working diagnosis was that her oesophageal varices were secondary to superior vena cava stenosis which was believed to be caused by her previous internal jugular permanent dialysis catheter which stayed in situ for around 12 months. Attempts to intervene with this stenosis were deemed unsafe because of the proximity of the stenosis to the right atrium and the risk of fatal bleeding it might carry. She was anticoagulated with low-molecular-weight heparin with regular endoscopic surveillance of her varices. Following this event, her right brachiocephalic fistula clotted as well, leaving her without a dialysis access. Therefore, we have resorted to a femoral permanent dialysis catheter. She had no further episodes of oesophageal variceal bleed since the initial episode.

\section{Discussion}

Central venous thrombosis and stenosis is not uncommon among the haemodialysis population given the need, in some patients, to start dialysis via permanent haemodialysis catheters. ${ }^{1-3}$ However, it is very rarely reported to cause bleeding oesophageal varices. In the case we are presenting, the presence of two functioning fistulae had led to progression into oesophageal bleeding given the extensive venous drainage into a blocked superior vena cava that encouraged the formation of extensive collaterals; the oesophageal varices are one of them. In this lady's case, it was technically difficult to treat the superior vena cava stenosis but the fact that her fistulae clotted relieved some of the back pressure on the varices and reduced future bleeding risk.

\section{Conflict of interest statement}

None declared.

\section{References}

1 Basye SL, Greenwell MW, Acchiardo SR, Parks FD, Wall BM. 9 bleeding oesophageal varices secondary to dialysis catheter induced superior vena cava stenosis. J Investig Med 2006;54:257.

2 Nayudu Sk, Dev A, Kanneganti K. "Downhill” esophageal varices due to dialysis catheter-induced superior vena caval occlusion: a rare cause of upper gastrointestinal bleeding. Case Rep Gastrointest Med 2013;2013:83079.

3 Bhandari D, Sachdeva M. Superior vena cava stenosis with proximal esophageal varices: a rare hemodialysis catheter related complication. Am J Kidney Dis 2014;63:A32. 